\title{
Why Do Some Goods Bear Higher Taxes than Others?*
}

\author{
MARTIN J. OSBORNE \\ Economics Department, Columbia University, New York, New York 10027
}

Received July 13, 1981; revised May 13, 1983

\begin{abstract}
Taxation in an economy containing land and labor is studied. The tax system is a compromise based on the relative power of all groups of individuals. (The model extends one of Aumann and Kurz.) In response to the threat of taxation by a majority, individuals can evade taxation of their labor-time (by destroying it), but cannot avoid taxation of the land they own. One result is that the compromise tax rate on land is higher than that on labor. This contrasts with the classical normative result that in order to minimize the efficiency loss, this tax rate should be higher. Journal of Economic Literature Classification Numbers: 022, 320.
\end{abstract}

\section{INTRODUCTION}

In recent years much attention has been given to the characteristics of the tax system a government should choose in order to meet a certain objective (typically, to maximize a social welfare function). To the extent that the characteristics are independent of the objective, this provides an explanation for the nature of the observed tax system. In general, however, the "optimal taxes" are quite sensitive to the objective. Thus we need to go further, and explain why a government chooses one objective rather than another.

A formal analysis of this question has recently been presented by Aumann and Kurz [2-4]. The basic idea behind their model is the following. The continued existence of a government depends on its ability to effectively control the economy. It can do so only if all the individuals and groups in the economy cooperate. If a group is capable of taking an action which disrupts the efforts of the government, and is not too costly to the group members, it will be tempted to do so in response to any policy which is not to its liking. Thus, in order to stay in power, a government has to weigh most heavily the interests of those groups which are most powerful in this

* Some of the material here is drawn from my dissertation [9], which was supported by National Science Foundation Grant SOC75-21820-A01 at the Institute for Mathematical Studies in the Social Sciences, Stanford University. 
sense. The resulting tax system is a compromise based on the power of all the groups in the economy.

Specifically, Aumann and Kurz assume that any majority can expropriate the endowment of its complement. This embodies the idea that in a democracy any majority can form a government and impose a wealth tax at any rate, including $100 \%$. A minority can evade such taxation by simply destroying its endowment-i.e. by "going on strike." Of course, in equilibrium these extreme actions are not carried out, but the fact that they are available determines the compromise which is reached. The outcome is an efficient allocation, which can thus be supported by setting prices and levying lump-sum taxes. Aumann and Kurz show that the tax an individual pays depends only on his utility function and the value of his endowment at the supporting prices; the marginal tax rate is always at least $50 \%$.

Here we investigate the effects of some variations in Aumann and Kurz' assumptions. The most significant change concerns the strategies available to a minority. Some goods-like labor-time - can indeed be "destroyed" by their owners. For other goods-like land-no such destruction is possible. The flow of land-services (e.g., the output of food) may be destroyed, but a majority can always expropriate the land and produce the land-services itself. We capture this essential difference between human and physical capital by assuming that there are two goods-one which can be destroyed ("labor"), and one which cannot be ("land"). We find that the tax an individual pays depends on his utility function and the values of his endowments of land and labor at the supporting prices; the marginal tax rate on land-wealth always exceeds that on labor-wealth. Further, the presence of land means that the marginal tax rate on labor may be less than $50 \%$.

The distinction between land and labor is of course a classical one. Also, there is a classical argument to the effect that if the objective is to raise a given tax revenue with a minimal loss of efficiency, the two goods should bear different taxes-the tax rate on land should be relatively high (since this does not affect supply), while that on labor-time should be relatively low. Our (positive) theory yields the same pattern of taxation, though for quite different reasons. Here, land is taxed heavily because its owners are in a weak position when it comes to issuing threats.

We depart from the assumptions of Aumann and Kurz in two other ways. First, we allow for the distribution of votes to be nonuniform. In other words, a group containing less than half the population may have more than half the votes, and hence be able to impose taxes on its complement. (This feature was first introduced by Aumann, Kurz, and Neyman [5].) We actually go further, and allow the voting weight of an individual to depend on his endowment, rather than simply being exogenous. (A wealthy group may be able to lobby for its interests more effectively than a poor one.) As one would expect, the higher an individual's voting weight, the lower his tax. 
If voting weight is an increasing concave function of endowments, then, at least in a class of examples, the tax system is progressive; if voting weight is a convex increasing function of endowments, then the tax system is regressive.

Our final departure from the assumptions of Aumann and Kurz concerns the definition of a majority. We allow the fraction of votes needed by a coalition in order to impose taxes to be any number at least equal to onehalf, rather than restricting it to one-half (see the discussion in Section 3). We find that the larger the critical fraction, the lower the marginal tax rates; if the critical fraction is high enough, both tax rates may be less than $50 \%$.

In Sections 2 through 5 we define and discuss the model. Our main result, which characterizes the compromise allocations of goods, and hence the taxes which "support" them, is established in Section 7, after some preliminary arguments in Section 6. In Sections 8 and 9 we discuss the characteristics of the predicted taxes.

\section{The EConomic Structure of Society}

The economic structure of society is that of an exchange economy. Each individual has an endowment of goods and a utility function. To simplify our arguments, we assume there are two goods" ("labor" and "land"), and a continuum of individuals of finitely many different types. The space of individuals is the measure space $(T, \mathscr{C}, \mu)$ (in which $(T, \mathscr{C})$ is isomorphic to $([0,1], \mathscr{B})$, where $\mathscr{B}$ is the $\sigma$-field of Borel subsets $)$. An element of $\mathscr{C}$ is a coalition; the population measure $\mu$ is nonnegative and nonatomic, and $\mu(T)=1$. (All measurability statements and integrations are with respect to $\mu$, unless otherwise noted.) The utility function of $t \in T$ is $u_{t}: \Omega \rightarrow \mathbb{R}$, where $\Omega=\mathbb{R}_{+}^{2}$, and $t$ 's endowment (density) is $\mathbf{e}(t)=(\mathbf{a}(t), \mathbf{b}(t)) \in \Omega$. The measurable partition $\left\{T_{1}, \ldots, T_{k}\right\}$ of $T$ delineates the $k$ types: for each $j=$ $1, \ldots, k$ there is a function $U_{j}: \Omega \rightarrow \mathbb{R}$ and a point $e_{j}=\left(a_{j}, b_{j}\right) \in \Omega$ such that $u_{t}=U_{j}$ and $^{2} \mathbf{e}(t)=e_{j}$ for all $t \in T_{j}$. We write functions on $T$ (like $\mathbf{e}$ ) in boldface; if $\mathbf{f}$ is such a function (and is measurable) and $S$ is a coalition, we write $\int_{S} \mathbf{f}$ instead of $\int_{S} \mathbf{f}(t) d \mu$, and $\int \mathbf{f}$ instead of $\int_{T} \mathbf{f}$. We call a function on $T$ which is constant on each $T_{j}$ a finite-type function. If $\mathrm{f}$ is such a function, we denote the associated vector $f$ (i.e., $\mathbf{f}(t)=f_{j}$ if $t \in T_{j}$ ), and vice versa. In this case we also write $f(S)$ instead of $\sum_{j=1}^{k} f_{j} \mu\left(S \cap T_{j}\right)\left(=\int_{S} \mathbf{f}\right)$. We assume that

\footnotetext{
${ }^{1}$ The main characterization result (Proposition 7.6) applies to an economy with many goods; as to the generalization of the "comparative static" results in Sections 8 and 9, see footnote 11 .

${ }^{2}$ Note that a "type" here is more restrictive than in [6], where individuals of the same type are not required to possess the same endowment.
} 
$\int \mathbf{e} \gg 0$ and $U_{j}(0)=0$ for $j=1, \ldots, k$. We also make the following standard ${ }^{3}$ assumptions.

(2.1) For each $j=1, \ldots, k, U_{j}$ is concave and continuous, increasing on int $\Omega$, and for $i=1,2$, the partial derivative $D_{i} U_{j}(x)$ exists and is continuous at each $x \in \Omega$ with $x_{i}>0$.

(2.2) For each $\varepsilon>0$ there exists $B \in \mathbb{R}$ such that for all $j=1, \ldots, k$, $U_{j}(x) \leqslant \varepsilon \sum_{i=1}^{2} x_{l}$ whenever $\sum_{i=1}^{2} x_{i} \geqslant B$.

An allocation is a measurable function $\mathbf{x}: T \rightarrow \Omega$ with $\int \mathbf{x} \leqslant e(T)$. An allocation $\mathbf{x}$ is efficient if there is no allocation $\mathbf{y}$ such that $u_{t}(\mathbf{y}(t))>u_{t}(\mathbf{x}(t))$ a.e.

Let $\mathbf{x}$ be an efficient allocation. Under our assumptions, we can associate with $\mathbf{x}$ a "supporting" price $p \in \mathbb{R}_{++}^{2}$ (i.e., $p$ is such that $\mathbf{x}(t)$ a.e. maximizes $u_{t}$ on $\left.\{x \in \Omega: p x \leqslant p \mathbf{x}(t)\}\right)$. Following Aumann and Kurz, we call such a price an efficiency price for $\mathbf{x}$. We can also associate with $\mathbf{x}$ a finite-type function $\lambda: T \rightarrow \mathbb{R}_{+}$, which assigns a "weight" to each individual, such that $\mathbf{x}$ maximizes the weighted sum of utilities $\int \lambda u(\mathbf{x})$ over all allocations (where $\lambda u(\mathbf{x})$ is the real-valued function on $T$ with values of the form $\left.\lambda(t) u_{t}(\mathbf{x}(t))\right)$. Obviously both $p$ and $\lambda$ retain these properties if they are multiplied by positive constants, so they may both be normalized. For our analysis in the sequel, the normalizations need to be related to each other. The most compact way of specifying these normalizations is to follow Aumann and Kurz and define $(\lambda, p)$ to be an efficiency pair for $\mathbf{x}$ if

$$
\mathbf{x}(t) \text { a.e. maximizes } \lambda(t) u_{t}(x)-p x \text { over } x \in \Omega \text {. }
$$

(If $\mathbf{x} \gg 0$ this of course implies that a.e. $\lambda(t) D u_{t}(\mathbf{x}(t))=p$, so that given $\lambda$, we are normalizing $p$ such that $\int \lambda(t) \sum_{i=1}^{2} D_{i} u_{t}(\mathbf{x}(t))=\sum_{i=1}^{2} p_{i}$.)

\section{The Political Structure of Society}

In the strategic game which underlies our model, the nature of the set of strategies available to each group of individuals depends on whether the group commands a majority of the votes. In a democratic society, votes are distributed evenly among the population. However, the amount of influence of each individual may not be the same. A wealthy pressure group may be able to make its point much more effectively, and thus gain larger concessions for its members, than a poor one. We incorporate this by assigning a voting weight $\mathbf{w}(t)$ to each $t \in T$. We assume that individuals of

${ }^{3}$ See [3]. Condition (2.2) guarantees that the coalitional games which arise possess asymptotic (Shapley) values. 
the same type have the same voting weight, ${ }^{4}$ and normalize so that $\int \mathbf{w}=1$; $w(S)$ is the fraction of the votes possessed by the coalition $S$. Later we consider situations where $w(S)$ depends on the wealth of $S$.

The coalition $S$ is a majority if $w(S)>\alpha$ (i.e., $S$ possesses more than the fraction $\alpha$ of the votes), where $\alpha \geqslant \frac{1}{2}$. We allow $\alpha$ to exceed $\frac{1}{2}$ in order to take into account the fact that while a group commanding barely more than half the votes may be able to influence a government to some extent, only a larger group can be effective. A group may need substantially more influence than its complement in order to persuade a government to adopt its recommendations. The coalition $S$ is a minority if $w(S)<1-\alpha$; if $1-\alpha \leqslant$ $w(S) \leqslant \alpha$ then $S$ is neither a majority nor a minority.

\section{The Strategies of Coalitions}

As in Aumann and Kurz [2,3] we do not need to specify all the strategies available to each coalition. For the solution concept we use, it is enough to assume that certain "extreme" actions are available. Thus we assume (as do Aumann and Kurz) that one strategy available to a majority $S$ is the expropriation of any goods which its complement $(T \backslash S)$ owns. This captures the idea that a majority can impose any taxes it wishes. In order to incorporate the idea that an individual can avoid such taxes on his endowment of land, we depart from Aumann and Kurz' assumption that a minority may destroy its endowment. If $S$ is a minority, we assume that its endowment $a(S)$ of the first good- "labor"-can be destroyed, while its endowment $b(S)$ of the second good- "land"- cannot be. If $S$ is neither a majority nor a minority, it can neither expropriate, nor be expropriated.

A consequence of these assumptions is that a majority can assure itself of the entire land-endowment of society, but cannot guarantee for itself more than its own endowment of labor. At an "equilibrium" of our model, extreme actions are not carried out, but the fact that they exist determines the compromise which is reached.

The economic and political structure of society, together with the strategies available to each group, defines the economy, which we denote by $E$.

\section{Optimal Threats and the Solution Concept for the Economy}

We use the same solution concept as do Aumann and Kurz [2, 3]. The idea behind it is quite simple. First, each group announces a strategy, with

${ }^{4}$ This amounts to assuming merely that there are finitely many voting weights, since the sets $T_{j}$ can be specified arbitrarily. 
the understanding that it is a threat which will be carried out in the event that no agreement is reached. Then an agreement is negotiated on the basis of the announced threats. Everyone knows that this procedure will be followed, and so each group chooses its threat in such a way as to maximize (given all other threats) the payoffs of its members in the negotiated agreement. The specific model of the outcome of negotiation which is used is the Shapley value.

Although this solution concept does not capture all the subtleties of multilateral negotiation in a clearly definitive fashion, it does possess some attractive qualitative features. In the case where utility is transferable, Selten $[10]$ has shown that the outcome is unique among those satisfying a number of appealing axioms. When utility is not transferable, the solution is less well supported. However, it has yielded interesting results in a number of applications (see [1], for example, in addition to the work of Aumann and Kurz), and there is no other solution which comes close to modeling the complexity of multilateral bargaining.

We shall not define the whole solution formally, since part of our argument runs parallel to that of Aumann and Kurz [2,3]. It is easy to show that the "extreme" actions specified in the previous section are optimal threats. That is, in order to maximize its negotiated payoff, a majority should threaten to expropriate the goods owned by its complement, and the latter should destroy all its labor.

Given the optimal threats, the compromise is determined by Shapley's [11] nontransferable-utility value of the coalitional game defined by the payoffs when the optimal threats are carried out. Precisely, let $\lambda: T \rightarrow \mathbb{R}$ be a positive, measurable finite-type function. We call $\lambda$ a comparison function, ${ }^{5}$ and interpret $\lambda(t)$ as the weight attached to individual $t$ 's utility. Let $q_{\lambda}(S)$ be the aggregate weighted utility of $S$ when the optimal threats are carried out. Then $q_{\lambda}: \mathscr{C} \rightarrow \mathbb{R}$ is a coalitional game. Let $\phi q_{\lambda}$ be the asymptotic ${ }^{6}$ (Shapley) value of $q_{\lambda}$. If $\lambda$ is such that there exists a finite-type allocation $\mathbf{x}$ such that ${ }^{7}$

$$
\left(\phi q_{\lambda}\right)(S)=\int_{S} \lambda u(\mathbf{x}) \quad \text { for each } \quad S \in \mathscr{C}
$$

\footnotetext{
${ }^{5}$ Note that the fact that $\lambda$ is of finite type means that for $j=1, \ldots, k$, we are assuming that the weight attached to each member of $T_{j}$ is the same-i.e, we are imposing a condition of "equal treatment." This assumption is quite inessential to our arguments, but significantly simplifies the presentation. So long as $\lambda$ takes on finitely many values, our main characterization result holds; since it does not seem possible to show that every equilibrium $\lambda$ is of finite type, there may exist value allocations which do not involve equal treatment.

${ }^{6}$ The asymptotic value is only one of the several ways of extending the Shapley value to games with a continuum of players. However (as a referee pointed out), it is particularly appealing here, since Neyman and Samet [8] show that it is the unique continuous value on $b v^{\prime} N A * p N A$, which is a space within which all our games lie (see [6]).

${ }^{7}$ Recall that $\lambda u(\mathbf{x})$ is the function on $T$ with values of the form $\lambda(t) u_{t}(\mathbf{x}(t))$.
} 
then $\mathbf{x}$ is a (finite-type) value allocation of $E$. Thus, $\lambda$ is an "equilibrium" comparison function if the payoffs associated with the Shapley value of $q_{\lambda}$ can be achieved without any transfers of utility; or, equivalently, $\mathbf{x}$ is a value allocation if the $\lambda$-weighted utilities associated with it are the payoffs in the Shapley value of $q_{\lambda}$.

Now, the Shapley value of a player is a weighted sum of the increments in the worth of each coalition caused by the addition of that player. In a game with a continuum of players, "most" coalitions are perfect samples of the population (i.e., they contain the same fraction of each type). Thus in order to characterize the value allocations of the economy $E$, we need to study the weighted sum of utilities $q_{\lambda}(S)$ of such coalitions when the optimal threats are carried out, and in particular how $q_{\lambda}(S)$ varies with small changes in the composition of $S$. We do so in the next section, which allows us (in Section 7) to characterize the value allocations of $E$.

\section{The Welfare of Coalitions; Subeconomies}

Throughout this section, we fix the comparison function $\lambda$. We say that $\left(m_{1}, \ldots, m_{k}\right)$ is the profile of the coalition $S$ if $S$ contains the measure $m_{j}$ of individuals of type $j$ (i.e., $\mu\left(S \cap T_{j}\right)=m_{j}$ ), for $j=1, \ldots, k$. The maximal weighted sum of utilities that $S$ can attain depends on its profile and the goods at its disposal. If $S$ has access to the vector of goods $c=\left(c_{1}, c_{2}\right)$ then this maximum is

$g_{\lambda}(m, c)=\max \left\{\sum_{j=1}^{k} m_{j} \lambda_{j} U_{j}\left(x_{j}\right): \sum_{j=1}^{k} m_{j} x_{j} \leqslant c, x_{j} \in \Omega\right.$ for $\left.j=1, \ldots, k\right\}$.

Let $\xi_{\lambda}(m, c)$ be the optimal value of the Lagrange multiplier on the constraint. Using arguments like those in the proof of Proposition 39.13 of [6] it is easy to show that if $(m, c) \gg 0$ then the derivatives of $g_{\lambda}$ are as follows: ${ }^{8}$

$$
D_{j} g_{\lambda}(m, c)= \begin{cases}\lambda_{j} U_{j}\left(x_{j}^{*}\right)-\xi_{\lambda}(m, c) x_{j}^{*} & \text { for } j=1, \ldots, k \\ \left(\xi_{\lambda}(m, c)\right)_{j-k} & \text { for } j=k+1, k+2,\end{cases}
$$

where $\left(x_{1}^{*}, \ldots, x_{k}^{*}\right)$ is a maximizer in (6.1). These derivatives have clear intuitive interpretations. If an individual of type $i$ joins $S$ then he is assigned the bundle $x_{i}^{*}$, and his net contribution to the social welfare of $S$ is his utility of $x_{i}^{*}$ less the "shadow value" of $x_{i}^{*}$; if $S$ has access to an extra unit of good $h$, then the maximized social welfare increases by the shadow price of $h$.

\footnotetext{
${ }^{8}$ For $j=k+1, k+2$ the result follows from the "envelope theorem."
} 
Now, for $0 \leqslant \theta \leqslant 1$, let a $\theta$-subeconomy of $E$, which we denote $\theta E$, consist of the fraction $\theta$ of each type of individual, with each coalition in $\theta E$ retaining its political and strategic characteristics as a coalition in $E$. Thus, the profile of the set of individuals in $\theta E$, which we denote $\theta T$, is $\left(\theta \mu\left(T_{1}\right), \ldots, \theta \mu\left(T_{k}\right)\right)$, and if $S \subset \theta T$ is a majority in $E$ (i.e., $\left.w(S)>\alpha\right)$ then it can assure itself the vector of goods $(a(S), b(T))$. If $\theta T$ is a majority in $E$ (i.e., $\theta>\alpha)$ and $x^{\theta}=\left(x_{1}^{\theta}, \ldots, x_{k}^{\theta}\right)$ is a maximizer in (6.1) for $m=$ $\left(\theta \mu\left(T_{1}\right), \ldots, \theta \mu\left(T_{k}\right)\right)$ and $c=(\theta a(T), b(T))$, then we say that $x^{\theta}$ maximizes $\lambda$ welfare in $\theta T$. If we define an efficiency pair in $\theta E$ in the natural way (requiring the maximization in (2.3) only for almost all $t \in \theta T$ ), it is easy to show that

(6.3) if $x^{\theta}$ maximizes $\lambda$-welfare in $\theta E, \theta>\alpha, m=\left(\theta \mu\left(T_{1}\right), \ldots, \theta \mu\left(T_{k}\right)\right)$, and $c=(\theta a(T), b(T))$, then $\left(\lambda, \xi_{\lambda}(m, c)\right)$ is an efficiency pair for $x^{\theta}$ in $\theta E$; in particular, $\xi_{\lambda}(m, c)$ is an efficiency price for $x^{\theta}$ in $\theta E$.

\section{A Characterization of the Value Allocations of $E$}

As discussed in Section 5, in order to characterize the value allocations of $E$ we need to calculate the Shapley value of the game $q_{\lambda}$, where $q_{\lambda}(S)$ is the maximal $\lambda$-welfare of $S$ in $E$ when the optimal threats are carried out. Now, for each coalition $S$, the magnitude $q_{\lambda}(S)$ can be expressed in terms of the function $g_{\lambda}$ introduced in the previous section (see (6.1)). Fix $\lambda$, let $\eta_{j}(S)=$ $\mu\left(S \cap T_{j}\right)$ (the measure of the members of $S$ of type $j$ ) and let $\eta(S)=$ $\left(\eta_{1}(S), \ldots, \eta_{k}(S)\right)$. Suppose that the optimal threats are carried out. If $w(S)>\alpha$ then $S$ has access to the vector of goods $(a(S), b(T))$; if $1-\alpha \leqslant$ $w(S) \leqslant \alpha$ then $S$ has access to $(a(S), b(S))$; and if $w(S)<1-\alpha$ then $S$ has access to $(0,0)$. Thus

$$
q_{\lambda}(S)=\left\{\begin{array}{lll}
0 & \text { if } \quad 0 \leqslant w(S)<1-\alpha \\
g_{\lambda}(\eta(S),(a(S), b(S))) & \text { if } \quad 1-\alpha \leqslant w(S) \leqslant \alpha \\
g_{\lambda}(\eta(S),(a(S), b(T))) & \text { if } \quad \alpha<w(S) \leqslant 1
\end{array}\right.
$$

In order to calculate the asymptotic value of $q_{\lambda}$ we decompose $q_{\lambda}$ into a sum of games of the form

$$
q(S)= \begin{cases}0 & \text { if } \quad 0 \leqslant w(S)<\delta \\ f(\gamma(S)) & \text { if } \quad \delta \leqslant w(S) \leqslant 1\end{cases}
$$

where $\gamma=\left(\gamma_{1}, \ldots, \gamma_{k}\right)$ is a nonnegative nonatomic vector measure on $(T, \mathscr{C})$ and $f: \mathbb{R}_{+}^{k} \rightarrow \mathbb{R}_{+}$. For such a game, the results of Aumann and Kurz 
(Proposition 13.1 of [3]) and Neyman (Theorem 4.1 of [7]) imply ${ }^{9}$ the following.

\section{Proposition 7.3 (Aumann and Kurz, Neyman). If}

(7.4) $f$ is concave, non-decreasing, and continuous, and for $i=1, \ldots, k$ the partial derivative $D_{i} f(x)$ exists and is continuous whenever $x_{i}>0$, then the game $q$ defined in (7.2) has an asymptotic value $\phi q$ given by

$$
\begin{array}{r}
(\phi q)(S)=f(\delta \gamma(T)) w(S)+\sum_{j=1}^{k} \gamma_{j}(S) \int_{\delta}^{1} D_{j} f(\theta \gamma(T)) d \theta \\
\quad \text { for each } \quad S \in \mathscr{C} .
\end{array}
$$

This result allows us to characterize the value allocations of $E$. For notational simplicity, let $r_{\lambda}(T)$ be the maximal $\lambda$-welfare in $E$ (i.e., $r_{\lambda}(T)=$ $\left.g_{\lambda}(\eta(T),(a(T), b(T)))\right)$ and let $v_{\lambda}(\theta T)$ be the maximal $\lambda$-welfare in $\theta E$ (i.e., $v_{\lambda}(\theta T)=g_{\lambda}(\theta \eta(T),(\theta a(T), b(T)))$ if $\left.\theta>\alpha\right)$. Then we have the following.

Proposition 7.6. A finite-type value allocation of $E$ exists, and $x=$ $\left(x_{1}, \ldots, x_{k}\right)$ is such an allocation if and only if it is efficient and, for $j=1, \ldots, k$,

$$
\begin{aligned}
& 2(1-\alpha) \lambda_{j} U_{j}\left(x_{j}\right)+(2 \alpha-1) p\left(x_{j}-e_{j}\right) \\
& \quad-\int_{\alpha}^{1}\left\{\lambda_{j} U_{j}\left(x_{j}^{\theta}\right)-p^{\theta} x_{j}^{\theta}+p_{1}^{\theta} a_{j}\right\} d \theta=\left(v_{\lambda}(\alpha T)-(2 \alpha-1) r_{\lambda}(T)\right) w_{j},
\end{aligned}
$$

where $(\lambda, p)$ is an efficiency pair for $x, x^{\theta}$ maximizes $\lambda$-welfare in $\theta E$, and $p^{\theta}=\left(p_{1}^{\theta}, p_{2}^{\theta}\right)$ is an efficiency price for $x^{\theta}$ in $\theta E$.

Proof. Existence is proved in the Appendix. To establish the characterization (7.7), we fix $\lambda$, decompose $q_{\lambda}$ into a sum of three games, and calculate the value of each separately. To define the decomposition, let $h_{\lambda}(\eta(S), a(S))=g_{\lambda}(\eta(S),(a(S), b(T)))$ for each coalition $S$. Then we have $q_{\lambda}=q_{\lambda}^{1}-q_{\lambda}^{2}+q_{\lambda}^{3}$, where each $q_{\lambda}^{i}$ is of type (7.2), with $\delta^{1}=1-\alpha$, $\delta^{2}=\delta^{3}=\alpha, f^{1}=f^{2}=g_{\lambda}, f^{3}=h_{\lambda}, \gamma^{1}=\gamma^{2}=(\eta, a, b)$ and $\gamma^{3}=(\eta, a)$. The additivity of the value means that

$$
\phi q_{\lambda}=\phi q_{\lambda}^{1}-\phi q_{\lambda}^{2}+\phi q_{\lambda}^{3} .
$$

The arguments of Aumann and Shapley (see ${ }^{10}$ Lemma 39.9 and Proposition 39.13 of [6]) establish (given (2.1) and (2.2)) that $g_{\lambda}$ and $h_{\lambda}$

\footnotetext{
'For the details, see Proposition 3.16 and Lemma 3.17 of [9].

${ }^{10} \mathrm{~A}$ small change needs to be made in the arguments, since in Lemma 39.9 it is assumed that each utility function is increasing, rather than merely nondecreasing.
} 
satisfy (7.4) for each $\lambda$. Their derivatives can be obtained from (6.2), so we can use (7.5) to write down the value of each game $q_{\lambda}^{i}, i=1,2,3$. First consider the cases $i=1,2$. Since $g_{\lambda}$ is homogeneous of degree one, and hence its derivatives are homogeneous of degree zero, we have $f^{i}\left(\delta^{i} \gamma^{i}(T)\right)=$ $\delta^{i} g_{\lambda}\left(\eta(T), \quad(a(T), b(T))=\delta^{i} r_{\lambda}(T), \quad\right.$ and $\quad D_{j} f^{i}\left(\theta \gamma^{i}(T)\right)=D_{j} g_{\lambda}\left(\gamma^{i}(T)\right)$ for $i=1$, 2. Hence, using $e(S)=\sum_{j=1}^{k} e_{j} \eta_{j}(S)$ and $w(S)=\sum_{j=1}^{k} w_{j} \eta_{j}(S)$, (7.5) and (6.2) give, for each coalition $S$,

$$
\left(\phi q_{\lambda}^{1}\right)(S)=\sum_{j=1}^{k} \eta_{j}(S)\left((1-\alpha) r_{\lambda}(T) w_{j}+\alpha\left(\lambda_{j} U_{j}\left(x_{j}\right)-p x_{j}+p e_{j}\right)\right)
$$

and

$$
\left(\phi q_{\lambda}^{2}\right)(S)=\sum_{j=1}^{k} \eta_{j}(S)\left(\alpha r_{\lambda}(T) w_{j}+(1-\alpha)\left(\lambda_{j} U_{j}\left(x_{j}\right)-p x_{j}+p e_{j}\right)\right),
$$

where $x=\left(x_{1}, \ldots, x_{k}\right)$ maximizes $\lambda$-welfare in $E$, and $p$ is an efficiency price for $x$ (using (6.3)). Now consider $q_{\lambda}^{3}$. We have $f^{3}\left(\delta^{3} \gamma^{3}(T)\right)=$ $h_{\lambda}(\alpha \eta(T), \alpha a(T))=v_{\lambda}(\alpha T)$, so

$$
\left(\phi q_{\lambda}^{3}\right)(S)=\sum_{j=1}^{k} \eta_{j}(S)\left\{v_{\lambda}(\alpha T) w_{j}+\int_{\alpha}^{1}\left(\lambda_{j} U_{j}\left(x_{j}^{\theta}\right)-p^{\theta} x_{j}^{\theta}+p_{1}^{\theta} a_{j}\right) d \theta\right\}^{\prime},
$$

where $x^{\theta}$ maximizes $\lambda$-welfare in $\theta E$, and $p^{\theta}$ is an efficiency price for $x^{\theta}$. Now we can combine (7.9), (7.10), and (7.11) in (7.8) to give an expression for $\left(\phi q_{\lambda}\right)(S)$. Finally we can use this and the fact that $\int_{s} \lambda u(\mathbf{x})=$ $\sum_{j=1}^{k} \eta_{j}(S) \lambda_{j} U_{j}\left(x_{j}\right)$ in (5.1) to obtain (7.7) (by setting $S=T_{j}$ for each $j=1, \ldots, k)$.

One can think of finding a value allocation in the following way. Choose an efficient allocation $x$, and find an efficiency pair $(\lambda, p)$ for it; given $\lambda$, find the $\lambda$-welfare maximizing $x^{\theta}$ for each subeconomy $\theta E$, and an efficiency price $p^{\theta}$ for each $x^{\theta}$. Then check to see if (7.7) is satisfied. If (and only if) it is, $x$ is a value allocation.

Although the form of (7.7) bears some similarity to the characterization obtained by Aumann and Kurz (see (9.15) of [3]), there is a significant qualitative difference. Under their assumptions, each subeconomy $\theta E$ is identical to $E$ except for the measure of the set of individuals in it. Thus, when all goods can be destroyed, a coalition can assure itself of no more than its own endowment (when the optimal threats are carried out), so in particular $\theta T$ can assure itself of precisely $\theta e(T)$. This means that if $x$ is an efficient allocation in $E$ then, restricted to $\theta T$, it is an efficient allocation in $\theta E$; if $x$ maximizes $\lambda$-welfare in $E$ then its restriction maximizes $\lambda$-welfare in $\theta E$, and the supporting prices are the same. When a good cannot be destroyed, a majority has access to the entire endowment of society of that good, so that 
the amount of that good available per head in $\theta E$ increases as $\theta$ falls (so long as $\theta T$ is a majority). This means that the subeconomies $\theta E$ do not resemble $E$, and an individual's worth depends not only on his wealth at the supporting prices in $E$, but also on his wealth at the supporting prices $p^{\theta}$ in each subeconomy ${ }^{11} \theta E$.

\section{COMPARISONS WITHIN AN ECONOMY}

The characterization (7.7) determines the bundle of goods $x_{j}$ which each individual of type $j$ receives in a value allocation, and hence the lump-sum taxes $p\left(e_{j}-x_{j}\right)$ which "support" this allocation. Here we study the characteristics of these taxes.

First we transform (7.7) into a more amenable form. For each type $j$ and for each $\alpha \leqslant \theta \leqslant 1$, let $V_{j}^{\theta}: \mathbb{R}_{+} \rightarrow \mathbb{R}$ be the indirect utility function associated with $U_{j}$ at the price $p^{\theta}$ given in Proposition 7.6. Let $Z_{j}^{\theta}=p^{\theta} x_{j}^{\theta}$, the value of $j$ 's bundle in the $\lambda$-welfare maximizing allocation $x^{\theta}$ in $\theta E$. As before, for $\theta=1$, we drop the superscript; $Z_{j}\left(=p x_{j}\right)$ is the post-tax wealth of $j$. Finally, let $A_{j}=p_{1} a_{j}$, the (pre-tax) labor-wealth of $j$, and $B_{j}=p_{2} b_{j}$, the (pre-tax) land-wealth of $j$. Now, since $p^{\theta}$ is an efficiency price for $x^{\theta}$ in $\theta E$ we have $U_{j}\left(x_{j}^{\theta}\right)=V_{j}^{\theta}\left(Z_{j}^{\theta}\right)$ and $^{12} \lambda_{j} D V_{j}^{\theta}\left(Z_{j}^{\theta}\right)=1$ for all $\alpha \leqslant \theta \leqslant 1$, for each $j=1, \ldots, k$. Thus we can write (7.7) as

$$
\begin{aligned}
2(1-\alpha) & \left(V_{j}\left(Z_{j}\right) / D V_{j}\left(Z_{j}\right)\right)+(2 \alpha-1) Z_{j}-\int_{a}^{1}\left(V_{j}^{\theta}\left(Z_{j}^{\theta}\right) / D V_{j}\left(Z_{j}\right)-Z_{j}^{\theta}\right) d \theta \\
= & \left(v_{\mathcal{\lambda}}(\alpha T)-(2 \alpha-1) r_{\mathcal{\lambda}}(T)\right) w_{j}+(2 \alpha-1)\left(A_{j}+B_{j}\right) \\
& +A_{j} \int_{\alpha}^{1}\left(p_{\mathrm{l}}^{\theta} / p_{1}\right) d \theta
\end{aligned}
$$

(The denominator of the second quotient is also equal to $D V_{j}^{\theta}\left(Z_{j}^{\theta}\right)$.)

Consider a set of individuals with the same utility function and voting

${ }^{11}$ For this reason, if there is more than one good of each type, the taxes which support a value allocation are not wealth taxes: the tax paid by an individual does not depend solely on the values of his endowments of the two types of goods at the price $p$, but also on their values at the supporting prices $p^{\theta}$ in the subeconomies. Unless $\theta E$ is similar to $E$ (as under the assumptions of Aumann and Kurz, or under the assumptions here when in addition the utility functions possess some homogeneity (see Section 9)), the values of an individual's endowments at the supporting price $p$ do not adequately measure his power, which is based on the contributions he can make in subeconomies. Thus, when there are many goods, the comparative static results of the next two sections which relate to the effect of changes in wealth on taxes need to be qualified.

${ }^{12}$ The fact that $(\lambda, p)$ is an efficiency pair for $x$ means that $1 / \lambda$, is the optimal value of the Lagrange multiplier in $j$ 's utility maximization problem (see (2.3)). 
weight. We shall study how the taxes paid by these individuals depend on their endowments. From (8.1), the post-tax wealth of one of these individuals depends on his labor- and land-wealths, say $Z_{j}=H\left(A_{j}, B_{j}\right)$. Since there is only a finite number of types in the model, the function $H$ is defined (via (8.1)) only at a finite number of points. However, by making a limiting argument in which the measure of each type goes to zero, we can justify taking the derivatives of $H$. Suppose the individuals in the set we are considering all have the utility function $U$ and voting weight $w$, and let the indirect utility functions be $V^{\theta}, \alpha \leqslant \theta \leqslant 1$. Then an individual in this set with labor- and land-wealths $A$ and $B$ has a post-tax wealth of $Z=H(A, B)$. Differentiating in (8.1) we find that for $i=1,2$,

$$
D_{i} H(A, B)=\frac{C_{i}}{1+G(Z)\left(2(1-\alpha)-\int_{\alpha}^{1}\left(V^{\theta}\left(Z^{\theta}\right) / V(Z)\right) d \theta\right)},
$$

where $C_{1}=(2 \alpha-1)+\int_{\alpha}^{1}\left(p_{1}^{\theta} / p_{1}\right) d \theta, \quad C_{2}=2 \alpha-1$, and $G(Z)=-V(Z) \times$ $D^{2} V(Z) /(D V(Z))^{2}$. We now argue that each derivative given in (8.2) is nonnegative (i.e., post-tax wealth is increasing in labor- and land-wealths, as one would expect). Consider two individuals with the same utility function, but different endowments. Suppose that $e_{j}>e_{h}$. Then if $j$ replaces $h$ in a coalition, the worth of that coalition in the game $q_{\lambda}$ increases, for any given comparison function $\lambda$. Thus, the marginal worth of $j$ exceeds that of $h$ in each coalition, so that the payoff of $j$ in the Shapley value of $q_{\lambda}$ also exceeds that of $h$. This is true in particular for the "equilibrium" $\lambda$, so $\lambda_{j} U\left(x_{j}\right)>$ $\lambda_{h} U\left(x_{h}\right)$, or $V\left(Z_{j}\right) / D V\left(Z_{j}\right)>V\left(Z_{h}\right) / D V\left(Z_{h}\right)$. But $V(Z) / D V(Z)$ is increasing (since $V$, like $U$, is concave), so that $Z_{j}>Z_{h}$. That is, post-tax wealth is increasing in endowments.

Let the tax paid by an individual with labor-and land-wealths $(A, B)$ be $\tau(A, B) \equiv A+B-H(A, B)$. We call $D_{i} \tau(A, B)=1-D_{i} H(A, B), i=1,2$, the marginal tax rates, even though no individual is confronted with a tax schedule, since the taxes are lump-sum; the marginal tax rates reflect the variation in taxes among individuals with different wealths. Since $D_{i} H(A, B) \geqslant 0$ for $i=1,2$, as argued above, we know the marginal tax rates are both at most 1 ; given that $C_{1}>C_{2},(8.2)$ implies that

the marginal tax rate on land-wealth exceeds that on labor-wealth.

Immediately from (8.2) we can also see that if $\alpha=\frac{1}{2}$-if a majority is precisely $50 \%$-then the marginal tax rate on land-wealth is $100 \%$, so that an individual's post-tax wealth is independent of his land endowment. If $\alpha$ exceeds $\frac{1}{2}$, then both marginal tax rates are less than $100 \%$, and may in fact be less than $50 \%$; even when $\alpha=\frac{1}{2}$, the marginal tax rate on labor-wealth may be less than $50 \%$ (for examples, see the next section). These last facts are significant because a striking feature of the tax rates under the 
assumptions of Aumann and Kurz ( $\alpha=\frac{1}{2}$, all goods can be destroyed) is that they always exceed $50 \%$. At least in a class of examples (see the next section), the presence of land lowers the tax rate on labor; an increase in $\alpha$ has the same effect.

As in the model of Aumann and Kurz, the tax rates depend on the "fear of ruin" $G(Z)$ at the post-tax wealth (see (5.6) of [2]). However, here the dependence is not as clearcut, since the tax rates also depend on the ratios $V^{\theta}\left(Z^{\theta}\right) / V(Z)$ for each $\alpha \leqslant \theta \leqslant 1$ (see (8.2)), and these are not systematically related to the fear of ruin. Thus the prominence of the latter in the determination of the tax rates in Aumann and Kurz' model is not inherent in the general framework. Rather, it comes from their specific assumptions on the strategic options of groups, which ensure that the subeconomies $\theta E$ are identical except for size (see the discussion at the end of the previous section).

Now consider how the taxes depend on voting weight. As before, since an individual with a higher voting weight contributes more to the worth of every coalition, his post-tax wealth is higher, and hence his tax, for each level of wealth, is lower. On the other hand, from (8.2) we can see that the marginal tax rates are independent of voting weight. Thus the tax schedule for individuals with high voting weight is similar to that for individuals with low voting weight, but is shifted down.

Finally, suppose that voting weight depends on endowments, say, $w_{j}=$ $f\left(a_{j}, b_{j}\right)$. From (8.1) we can derive an expression like (8.2); the only difference is that the numerator changes from $C_{i}$ to $C_{i}+\left(v_{\lambda}(\alpha T)-\right.$ $\left.(2 \alpha-1) r_{\lambda}(T)\right) D_{i} f\left(A / p_{1}, B / p_{2}\right) / p_{i}$ for $i=1,2$. Now $v_{\lambda}(\alpha T)>\alpha r_{\lambda}(T)>$ $(2 \alpha-1) r_{\lambda}(T)$, so if $f$ is increasing and concave (i.e., a higher endowment conveys more, but not proportionately more power), this numerator is decreasing in labor-and land-wealths $A$ and $B$. Thus if the denominator in (8.2) is independent of $Z$ (as it is under the assumptions in the next section), the tax system is progressive. On the other hand, if $f$ is increasing and convex (because, for example, there are increasing returns to wealth in terms of lobbying effectiveness) then the tax system is regressive. Since the sum of the taxes is zero, this means that in this latter case, individuals with low wealth pay taxes, while those with high wealth receive subsidies.

\section{Comparisons between Economies: A Class of Examples}

Here we study how the tax system varies with some aggregate characteristics of the economy. For example, we consider how taxes change with the fraction $\alpha$ which constitutes a majority, and with the degree of risk aversion of all individuals. Without making more specific assumptions than previously, it does not seem possible to pin down these changes. Suppose 
some parameter in the economy changes. Then in order to know how the set of value allocations changes, we need to know how the solutions to (7.7) change. This means that we need to know how the efficiency pair varies with the allocation, and how the change in the efficiency weights $\lambda$ causes the $\lambda$ welfare maximizing allocation $x^{\theta}$ and the supporting prices $p^{\theta}$ to change, for each $\theta$. For an arbitrary collection of utility functions, these changes can be quite irregular.

It helps to assume that for each fixed quantity of labor $y$, each utility function $U_{j}(y, \cdot)$ is homogeneous of the same degree in land, so that $U_{j}(y, z)=F_{j}(y) z^{\omega}$, for some $F_{j}$ and some $0<\omega<1$. Then if $x=(y, z)$ maximizes $\lambda$-welfare in $E$, it is easy to check that $x^{\theta}=(y, z / \theta)$ maximizes $\lambda$ welfare in $\theta E$; if $p=\left(p_{1}, p_{2}\right)$ is an efficiency price for $x$ then $p^{\theta}=$ $\left(\theta^{-\omega} p_{1}, \theta^{1-\omega} p_{2}\right)$ is an efficiency price for $x^{\theta}$ in $\theta E$. Thus, in the notation of the previous section, $V_{j}^{\theta}\left(Z_{j}^{\theta}\right)=U_{j}\left(x_{j}^{\theta}\right)=\theta^{-\omega} U_{j}\left(x_{j}\right)$ for each $\alpha \leqslant \theta \leqslant 1$. Substituting these relations into (8.2) and using the fact that the marginal tax rate $D_{i} \tau(A, B)$ on good $i$ equals $1-D_{i} H(A, B)$, we have, for $i=1,2$,

$$
D_{i} \tau(A, B)=1-\frac{C_{i}}{1+(1-\zeta-\delta) G(Z)}
$$

where $\delta=\left(1-\alpha^{1-\omega}\right) /(1-\omega), \zeta=2 \alpha-1, C_{1}=\delta+\zeta$, and $C_{2}=\zeta$.

This homogeneity assumption means that behavior in the subeconomies is systematically related to behavior in the whole economy. However, it does not allow us to compare tax rates in different economies, unless we can say how the fear of ruin $G(Z)$ of the indirect utility function at a value allocation differs in the economies. An additional assumption which makes comparisons possible is that the fear of ruin of the indirect utility function is independent of both price and wealth. That is, $G(Z)$ is a constant, independent of $p$ and $Z$. This implies ${ }^{13}$ that the indirect utility function $V$ is homogeneous of some degree $\beta \in(0,1)$, for all prices, which implies that the utility function itself is homogeneous of degree $\beta$. Given the previous homogeneity assumption, this means that $U_{j}$ is Cobb-Douglas. In this case, $G(Z)=(1-\beta) / \beta$ for all $Z$ and all prices, so that the tax rates are constant (independent of land- and labor-wealths). Letting the tax rate on labor-wealth be $\kappa_{1}$, and that on land-wealth $\kappa_{2}$, we have

$$
\kappa_{1}=1-\frac{\beta(\zeta+\delta)}{1-\gamma(\zeta+\delta)} \quad \text { and } \quad \kappa_{2}=1-\frac{\beta \zeta}{1-\gamma(\zeta+\delta)},
$$

where $\gamma=1-\beta$. It is easy to check that both these tax rates are decreasing functions of $\alpha$. Thus, 
among individuals with the same Cobb-Douglas utility function, the marginal tax rates are lower, the higher the fraction of the population which constitutes a majority.

Intuitively, when $\alpha$ is high it is more difficult to reach a consensus on redistribution, so the compromise tax rates are lower.

If both goods can be destroyed, then a small generalization ${ }^{14}$ of the results of Aumann and Kurz shows that, among individuals with the same CobbDouglas utility function, with overall degree of homogeneity $\beta=1-\gamma$, the tax rate on both goods is $(1-\alpha) /(1-\gamma \alpha)$. It is easy to show that this lies between $\kappa_{1}$ and $\kappa_{2}$. Thus, as one might expect, the presence of land lowers the tax rate on labor. It is also easy to check that the marginal tax rate $\kappa_{1}$ on labor can be less than $50 \%$ even if $\alpha=\frac{1}{2}$, as claimed in the previous section, and both tax rates can be less than $50 \%$ if $\alpha$ is large enough.

\section{APPENDiX: Existence of a Value Allocation}

Shapley [11, pp. 261-262] proves the following result, in which $A=$ $\left\{\lambda \in \mathbb{R}^{n}: \lambda \geqslant 0\right.$ and $\left.\sum_{i=1}^{n} \lambda_{i}=1\right\}$.

Theorem. Let $F: \Lambda \rightarrow \mathbb{R}^{n}$ be an upper-semicontinuous set-valued function which has nonempty, convex, and compact values. Let $f: \Lambda \rightarrow \mathbb{R}^{n}$ be continuous, with $\sum_{i=1}^{n} f_{i}(\lambda)=\max \left\{\sum_{i=1}^{n} z_{i}: z \in F(\lambda)\right\}$ for each $\lambda \in \Lambda$, and such that $f_{i}(\lambda) \geqslant 0$ whenever $\lambda_{i}=0$. Then there exists $\lambda \in A$ such that $f(\lambda) \in F(\lambda)$.

This result can be applied to establish the existence part of Proposition 7.6. Let $n=k$ (the number of types), and for each $\lambda \in A$, let $F(\lambda)=\left\{\left(y_{1}, \ldots, y_{k}\right): \quad y_{j}=\lambda_{j} U_{j}\left(x_{j}\right) \mu\left(T_{j}\right)\right.$ for some $\left(x_{1}, \ldots, x_{k}\right)$ such that $\left.\sum_{j=1}^{k} \mu\left(T_{j}\right) x_{j} \leqslant e(T)\right\}$. For each $\lambda \in A$, let $f_{j}(\lambda)=\left(\phi q_{\lambda}\right)\left(T_{j}\right)$ (where $q_{\lambda}$ is the game defined in (7.1)). Then $\sum_{j=1}^{k} f_{j}(\lambda)=\sum_{j=1}^{k}\left(\phi q_{\lambda}\right)\left(T_{j}\right)=\left(\phi q_{\lambda}\right)(T)=$ $q_{\lambda}(T)=\max \left\{\sum_{j=1}^{k} z_{j}: z \in F(\lambda)\right\}$ (using the efficiency of the value). Also, $q_{\lambda}$ is monotonic, so $\left(\phi q_{\lambda}\right)(S) \geqslant 0$ for every $S \in \mathscr{C}$, for any $\lambda$, so that certainly $f_{j}(\lambda) \geqslant 0$ if $\lambda_{j}=0$. Furthermore, $F$ and $f$ satisfy the continuity and convexity assumptions (given (2.1)). Thus there exists $\lambda \in A$ such that $\left(\phi q_{\lambda}\right)\left(T_{j}\right)=$ $\lambda_{j} U_{j}\left(x_{j}\right) \mu\left(T_{j}\right)$ for $j=1, \ldots, k$, for some $\left(x_{1}, \ldots, x_{k}\right)$ such that $\sum_{j=1}^{k} \mu\left(T_{j}\right) x_{j} \leqslant e(T)$. Given the symmetry of the value and the fact that all members of $T_{j}$ are identical, we have $\left(\phi q_{\lambda}\right)(S)=\int_{S} \lambda u(\mathbf{x})$ for each coalition $S$. Finally, we can argue as in [3, pp. 228-229] that under our assumptions $\lambda_{j}>0$ for $j=1, \ldots, k$, so that $\lambda$ is in fact a comparison function. This completes the demonstration.

\footnotetext{
${ }^{14}$ A generalization is needed, since Aumann and Kurz consider only the case where $a=\frac{1}{2}$.
} 


\section{ACKNOWLEDGMENTS}

I am greatly indebted to Mordecai Kurz, my disseration advisor, for innumerable suggestions and continuous encouragement. I should also like to thank Robert Aumann, Sergiu Hart, Haruo Imai, Abraham Neyman, and Yair Tauman for providing me with a wealth of ideas and a great deal of technical advice. In addition, Birgit Grodal, referees, and an Associate Editor. provided helpful comments.

\section{REFERENCES}

1. R. J. AumanN, Values of markets with a continuum of traders, Econometrica 43 (1975), 611-646.

2. R. J. Aumann And M. Kurz, Power and taxes, Econometrica 45 (1977), 1137-1161.

3. R. J. Aumann and M. Kurz, Power and taxes in a multi-commodity economy, Israel J. Math. 27 (1977), 185-234.

4. R. J. Aumann And M. Kurz, Power and taxes in a multi-commodity economy (updated), J. Pub. Econ. 9 (1978), 139-161.

5. R. J. Aumann, M. Kurz, and A. Neyman, "Public Goods and Power," Technical Report 273, Economics Series, Institute for Mathematical Studies in the Social Sciences, Stanford University, 1980.

6. R. J. Aumann and L. S. Shapley, "Values of Non-atomic Games," Princeton Univ. Press, Princeton, N.J., 1974.

7. A. Neyman, Singular games have asymptotic values, Math. Oper. Res. 6 (1981), 205-212.

8. A. Neyman AND D. SAMET, The existence and uniqueness of the value for some classes of non-atomic games, draft, 1981.

9. M. J. Osborne, "An Analysis of Power in Exchange Economies," Technical Report 291, Economics Series, Institute for Mathematical Studies in the Social Sciences, Stanford University, 1979.

10. R. Selten, Valuation of $n$-person games, in "Advances in Game Theory" (M. Dresher, L. S. Shapley, and A. W. Tucker, Eds.), pp. 577-626, Princeton Univ. Press, Princeton, N.J., 1964.

11. L. S. Shapley, Utility comparison and the theory of games, in "La Décision" (G. T. Guilbaud, Ed.), pp. 251-263, Editions du Centre National de la Recherche Scientifique, Paris, 1969. 